\title{
New roles for Notch in tuberous sclerosis
}

\author{
Warren S. Pear \\ Department of Pathology and Laboratory Medicine, Abramson Family Cancer Research Institute, University of Pennsylvania, Philadelphia, Pennsylvania, USA.
}

\begin{abstract}
Tuberous sclerosis complex (TSC) is a dominantly inherited disease that is characterized by the growth of multiple benign tumors that are often difficult to treat. TSC is caused by mutations that inactivate the TSC1 or TSC2 genes, which normally function to inhibit activation of mammalian target of rapamycin signaling. In this issue of the JCI, two studies reported by Karbowniczek et al. and Ma et al. link TSC inactivation with activated Notch signaling (see the related articles beginning on pages 93 and 103, respectively). Using a variety of approaches, both studies show that inactivation of TSC leads to Notch1 activation. Furthermore, studies in tumor cells suggest that inhibiting Notch slows growth of the tumor cells. Although much remains to be learned about the precise mechanisms by which TSC loss leads to Notch activation, the newly identified link of TSC to Notch provides the rationale for testing Notch inhibitors in TSC-associated tumors.
\end{abstract}

Tuberous sclerosis complex (TSC) is a dominantly inherited multisystem disease that is characterized by the formation of benign tumors in multiple organs $(1,2)$. The clinical manifestations of TSC are variable but frequently involve the nervous system, as evidenced by the occurrence of seizures and autism, which may, in part, result from benign growths ("tubers") in the cerebral cortex. Patients frequently develop tumors outside of the CNS; the development of renal angiomyolipomas (benign renal neoplasms composed of fat, vascular, and smooth muscle) and pulmonary lymphangioleiomyomatosis (LAM; abnormal proliferation of smooth muscle cells throughout lung interstitium) can lead to severe clinical problems that respond poorly to current treatments. TSC is caused by mutations that inactivate either the TSC1 or TSC2 genes, also known as hamartin and tuberin, respectively (3). TSC1 and TSC2 heterodimerize to inhibit the Ras homolog Rheb (Ras homo$\log$ enriched in brain), which functions to activate the mammalian target of rapamycin (mTOR) complex 1 (TORC1) (4) (Figure 1). Thus, inactivation of TSC1 or TSC2 leads to prolonged Rheb signaling and activation of the rapamycin-sensitive TORC $1 \mathrm{arm}$ of the mTOR signaling pathway. mTOR integrates a variety of signals induced by growth factor stimulation, hypoxia, and nutrient availability to control cell cycle, nutrient uptake, and transcription and translational control (5). Important downstream targets of mTOR are

Conflict of interest: W.S. Pear owns stock in Pfizer Inc.

Citation for this article: J. Clin. Invest. 120:84-87 (2010). doi:10.1172/JCI41897. ribosomal protein $\mathrm{S} 6$ kinase polypeptide 1 (S6K1) and eukaryotic translation initiation factor $4 \mathrm{e}$-binding protein 1 (4E-BP1) that regulate ribosome recruitment and protein translation. mTOR is activated in a wide variety of human tumors and is considered to be an excellent target for anticancer therapy (6). The identification of the MTOR pathway as a major player in the pathogenesis of TSC suggested that specific inhibition of this pathway may substantially benefit TSC patients (7). The data are sobering thus far, as treatment of TSC patients with the mTOR inhibitor sirolimus has induced only mild, transient improvement (8). These data suggest that better and/or additional therapeutic agents are needed to treat TSC. In this issue of the JCI, Karbowniczek et al. (9) and Ma et al. (10) report that TSC loss is associated with upregulation of the Notch signaling pathway. Therapeutic Notch inhibitors have already been developed, and related findings suggest that these inhibitors may be beneficial for the treatment of TSC.

TSC loss promotes Rheb-dependent Notch activation in flies, rodents, and humans

Inappropriate expression of lineage markers is a frequent characteristic of TSC tumors; CNS tubers express both neuronal and glial lineage markers, and both LAM and renal angiomyolipomas express smooth muscle and melanocytic markers. Moreover, cells within the clonal renal angiomyolipomas exhibit developmental plasticity, with the capacity to develop into fat, smooth muscle, and vascular lineage cells (11). These obser- vations served as the initial impetus for both Karbowniczek et al. (9) and Ma et al. (10) to explore the potential role of Notch signaling in this process, as Notch is an important arbiter of cell fate decisions (12). Using different model systems and approaches, both groups came to the same conclusion: TSC loss leads to Notch activation. Karbowniczek and coworkers (9) took advantage of the evolutionary conservation of the TSC pathway to study the function of TSC signals in the well-described differentiation of the sensory organ precursor (SOP) cell into the external sensory organ (ESO) of the fruit fly Drosophila melanogaster (13) (Figure 2A). TSC signaling is conserved from Drosophila to humans; for example, the Drosophila orthologs of the mammalian tuberous sclerosis proteins, dTSC1 and dTSC2, function downstream of the insulin receptor to regulate mTOR signals and cell size (14). Differentiation of the SOP cell into the ESO results from multiple asymmetric divisions that depend on differential regulation of Notch signaling to ultimately give rise to the differentiated ESO, which is comprised of an external bristle cell and socket cell as well as an internal sensory neuron and sheath cell (Figure 2A) (15). This process begins when the primary progenitor (pI) cell undergoes an asymmetric cell division to give rise to an anterior $\mathrm{PIIb}$ cell and a posterior pIIa cell. The Notch inhibitor Numb is asymmetrically distributed to the pIIb cell, such that Notch signaling persists in the pIIa cell, whereas it is inhibited in the pIIb cell. In the absence of Notch signaling, two pIIb cells are produced, whereas constitutive Notch signals lead to the production of two pIIa cells at the expense of pIIb differentiation. The latter results in duplication of the external bristle and socket cells, causing the appearance of extra microbristles on the flies. Karbowniczek et al. generated flies with loss-of-function TSC alleles and found that the SOP differentiation phenotypes were similar to Notch gain-of-function phenotypes (i.e., duplication of the pIIa cell resulting in extra microbristles) (Figure 2B). Furthermore, overexpression of Rheb recapitulated these phenotypes, 


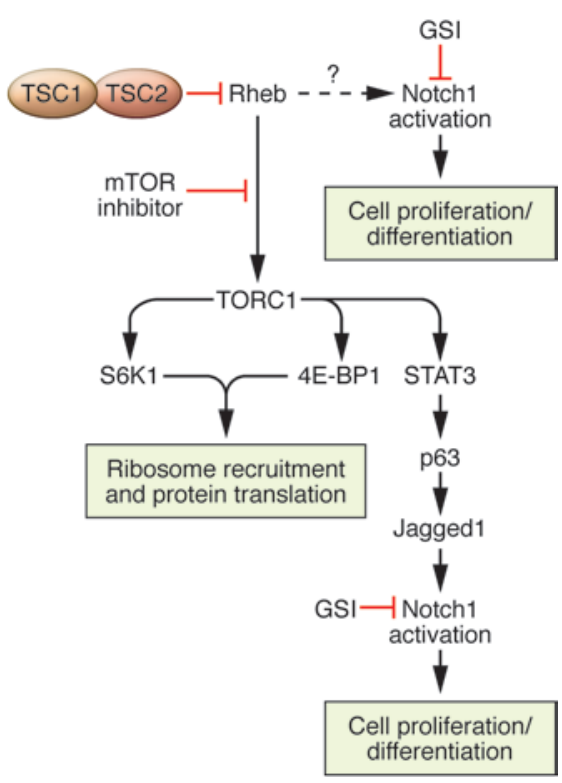

substantiating it as a TSC-inhibited target and providing additional evidence for a link to Notch signaling. The TSC effects on Notch appear to be cell context-specific, as the effects of TSC loss did not influence other Notch-related phenotypes in the fly, such as those described in fly wing differentiation.

To relate the Drosophila studies to humans, Karbowniczek and colleagues looked for evidence of Notch activation in tumors from TSC patients (9). In four renal angiomyolipomas from a single patient and several sporadic renal angiomyolipomas, they found increased levels of Notch1, the Notch ligand Delta-like 1 , and the Notch transcriptional target hairy and enhancer of split 1 (HES1) compared with control normal kidney. As with many tumor studies, it is difficult to know whether this indicates specific activation of Notch signaling in the tumor or normal Notch signaling in the cell type from which the clonal tumor was derived. To address the relevance of this finding to TSC, they investigated Notch signaling in a biallelic TSC2-deficient renal angiomyolipoma cell line. Both Rheb siRNA and exogenous expression of TSC2 inhibited the expression of the Notch targets HES1 and hairy/enhancer-of-split related with YRPW motif 1 (HEY1) to a similar extent as a $\gamma$-secretase inhibitor (GSI) that functions to inhibit Notch signaling. Moreover, treatment of xenografted rat TSClike tumors with a GSI inhibited tumor growth by more than $50 \%$ relative to vehicle control. Together these data offer the

\section{Figure 1}

Potential functions of Notch downstream of TSC. This schematic illustrates the potential mechanisms of Notch activation proposed by Karbowniczek et al. (9) and Ma et al. (10) in this issue of the JCI. TSC1 and TSC2 heterodimerize to inhibit activation of the Rheb-GTPase. In the absence of TSC1 or TSC2, Rheb is constitutively active, which leads to Notch activation by a TORC1-independent mechanism, as suggested by Karbowniczek et al. (9), and/or a TORC1dependent mechanism, as suggested by Ma et al. (10). The TORC1-independent mechanism of Notch1 activation remains to be elucidated, as indicated by the dotted line and question mark. In the TORC1-dependent pathway described by Ma et al., TORC1 activates STAT3, which leads to p63 upregulation of the Notch ligand Jagged1 and Notch1 activation. Notch is likely to influence cell differentiation and proliferation; however, it may also have effects on cell survival, cellular metabolism, and other cellular processes. Additional, important TORC1 targets are ribosomal protein S6 kinase polypeptide 1 (S6K1) and eukaryotic translation initiation factor 4e-binding protein 1 (4E-BP1), which have important functions in ribosome recruitment and protein translation. mTOR inhibitors act on TORC1; Notch inhibitors, such as GSI, act to prevent Notch signaling. It remains to be elucidated whether Notch activation occurs by a cellautonomous or a non-cell-autonomous mechanism in TSC.

possibility that TSC-related tumors activate Notch signaling and may be sensitive to Notch inhibitors.

TSC/Rheb signaling results in mTOR activation. Using a variety of approaches to inhibit mTOR signals, Karbowniczek and colleagues found that Notch remained active in the presence of $\mathrm{mTOR}$ inhibitors (9). These data suggest that the TSC/Rheb/Notch pathway is TORC1 independent. As TSC-dependent tumors have shown some responses to TORC1 inhibitors, the finding that Notch activation is TORC1 independent suggests that TORC1 and Notch inhibitors may have synergistic effects in treating TSCdependent tumors. Karbowniczek and colleagues propose that Notch signaling primarily influences the differentiation of TSC-related tumors, whereas mTOR promotes their proliferation. It remains to be seen whether these specialized roles exist in TSC tumors, as Notch has been shown to regulate mTOR, cell survival, and proliferation in Notch-dependent $\mathrm{T}$ cell acute lymphoblastic leukemia (16).

\section{mTOR activates Notch signaling in TSC-deficient mouse embryonic fibroblast cells and multiple human tumors}

In the study reported in this same issue by $\mathrm{Ma}$ and colleagues (10), the authors observed that TSC1- or TSC2-deficient mouse embryonic fibroblasts (MEFs) failed to differentiate into muscle or fat cells when exposed to constitutive expression of the master regulator of muscle differ- entiation (i.e., MyoD) or adipocyte differentiation (i.e., PPAR $\gamma$ ). Differentiation was restored in these cells by treatment with the mTOR inhibitor rapamycin, suggesting that elevated mTOR signals prevented differentiation of the TSC-deficient MEF cells. Because Notch signaling is an important regulator of myocyte and adipocyte differentiation, the authors initiated studies to determine the influence of TSC loss on Notch signaling. They found evidence of activated Notch 1 and upregulated HES1 transcription in TSC2-deficient MEF cells and also showed that HES1 expression is increased in renal tumor cells from a murine TSC model. In addition, the Notch ligand Jagged 1 was upregulated in the TSC-deficient MEF cells by a mechanism that may involve TORC1 upregulation of STAT3 that leads to p63 activation of Jagged1 (Figure 1). Surprisingly, Jagged 1 does not appear to be upregulated in the TSC-mutated human lung LAM tissues that they analyzed; instead, Jagged 2 is expressed in these cells. Although celltype specificity cannot be ruled out, additional experiments are required to explain this potential discrepancy, especially with regards to the TORC1-dependent mechanism that they propose. In contrast to the studies of Karbowniczek and colleagues (9), who found that Notch activation was TORC1 independent, Ma et al. found that both Jagged 1 expression and Notch 1 signaling were rapamycin sensitive in the TSC-deficient MEFs. In addition, they also found upregulated Jagged 1 expression in both PTEN-deficient and activated, AKT- 
A

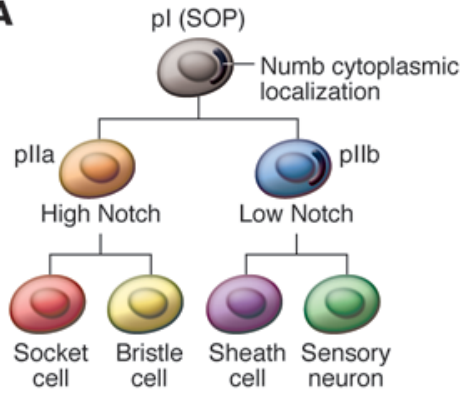

B

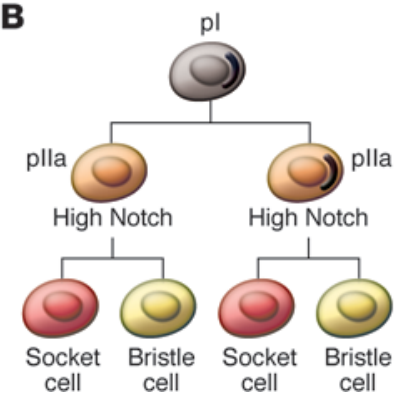

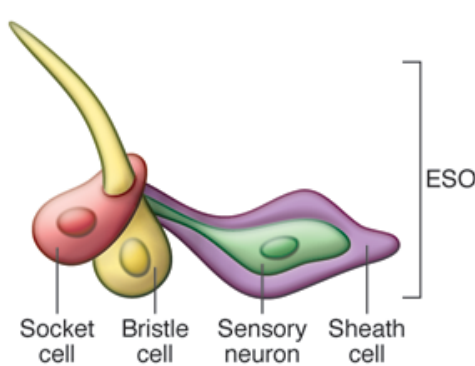

cell

\section{Summary}

Together, the work from both Karbowniczek et al. (9) and Ma et al. (10) provides strong evidence that Notch signaling is activated as a consequence of TSC loss. The work of Ma and coworkers presents tantalizing evidence that Notch activation may be a common event in mTOR-expressing tumors. Nevertheless, most of their data were derived from biochemical studies in cell lines, and it remains to be determined whether Notch inhibition can exert important antineoplastic effects on these tumors. Although both the Karbowniczek et al. (9) and Ma et al. (10) studies report that TSC loss leads to Notch activation, the mechanisms in each case appear quite different; the Karbowniczek et al. study proposes that Notch activation is mTOR independent, whereas the Ma et al. study suggests that it is mTOR dependent. The difference between these models may have important clinical implications, as one proposes that Notch inhibitors function independently of mTOR inhibitors, whereas the other proposes that the two agents act on the same pathway. The precise reasons for the different conclusions are not clear; however, they may result from studying the effects of TSC loss in different model systems. Additional studies are clearly warranted to determine the mechanism of Notch activation and whether this occurs by a single pathway or multiple pathways. Another issue that awaits further study is the mechanism by which Notch influences TSC pathogenesis. Both studies identify HES and HEY as potential Notch targets, but neither of these are Notch-specific targets. It is anticipated that future studies will identify additional targets that have the potential to serve both as biomarkers and therapeutic targets.

In summary, the two studies in this issue of the JCI provide compelling new evidence that identifies what I believe to be a novel role for Notch signaling in the pathogenesis of TSC and possibly other tumors. Although much remains to be learned about the mechanism and significance of this association, the new findings will undoubtedly catalyze these studies. Moreover, these findings may soon have clinical applications, as Notch inhibitors are currently being tested in clinical trials as anticancer agents. As TSC-associated tumors are difficult to treat, the potential to treat with a new class of compounds is exciting. 


\section{Acknowledgments}

I apologize to authors whose work could not be cited due to space limitations. Work in W.S. Pear's laboratory is supported by grants from the NIH and the Leukemia and Lymphoma Society.

Address correspondence to: Warren S. Pear, Department of Pathology and Laboratory Medicine, Abramson Family Cancer Research Institute, University of Pennsylvania, Philadelphia, Pennsylvania 19104-6160, USA. Phone: (215) 573-7764; Fax: (215) 5736875; E-mail:wpear@mail.med.upenn.edu.

1. Curatolo P, Bombardieri R, Jozwiak S. Tuberous sclerosis. Lancet. 2008;372(9639):657-668.

2. Crino PB, Nathanson KL, Henske EP. The tuberous sclerosis complex. $N$ Engl J Med. 2006;355(13):1345-1356.
3. Inoki K, Guan KL. Tuberous sclerosis complex, implication from a rare genetic disease to common cancer treatment. Hum Mol Genet. 2009;18(R1):R94-R100.

4. Huang J, Manning BD. The TSC1-TSC2 complex: a molecular switchboard controlling cell growth. Biochem J. 2008;412(2):179-190.

5. Sarbassov DD, Ali SM, Sabatini DM. Growing roles for the mTOR pathway. Curr Opin Cell Biol. 2005;17(6):596-603.

6. Guertin DA, Sabatini DM. Defining the role of mTOR in cancer. Cancer Cell. 2007;12(1):9-22.

7. Plas DR, Thomas G. Tubers and tumors: rapamycin therapy for benign and malignant tumors. Curr Opin Cell Biol. 2009;21(2):230-236.

8. Bissler JJ, et al. Sirolimus for angiomyolipoma in tuberous sclerosis complex or lymphangioleiomyomatosis. NEngl J Med. 2008;358(2):140-151.

9. Karbowniczek M, et al. The evolutionarily conserved TSC/Rheb pathway activates Notch in tuberous sclerosis complex and Drosophila external sensory organ development. J Clin Invest. 2010;120(1):93-102.

10. Ma J, et al. Mammalian target of rapamycin regulates murine and human cell differentiation through STAT3/p63/Jagged/Notch cascade. J Clin Invest. 2010;120(1):103-114.

11. Karbowniczek M, Yu J, Henske EP. Renal angiomyolipomas from patients with sporadic lymphangiomyomatosis contain both neoplastic and non-neoplastic vascular structures. Am J Pathol. 2003;162(2):491-500.

12. Artavanis-Tsakonas S, Rand MD, Lake RJ. Notch signaling: cell fate control and signal integration in development. Science. 1999;284(5415):770-776.

13. Posakony JW. Nature versus nurture: asymmetric cell divisions in Drosophila bristle development. Cell. 1994;76(3):415-418.

14. Potter CJ, Pedraza LG, Huang H, Xu T. The tuberous sclerosis complex (TSC) pathway and mechanism of size control. Biochem Soc Trans. 2003;31(Pt 3):584-586.

15. Roegiers F, Jan YN. Asymmetric cell division. Curr Opin Cell Biol. 2004;16(2):195-205.

16. Palomero T, Ferrando A. Oncogenic NOTCH1 control of MYC and PI3K: challenges and opportunities for anti-NOTCH1 therapy in T-cell acute lymphoblastic leukemias and lymphomas. Clin Cancer Res. 2008;14(17):5314-5317.

\title{
Oxidant stress derails the cardiac connexon connection
}

\author{
Gordon F. Tomaselli
}

Department of Medicine, Johns Hopkins University School of Medicine, Baltimore, Maryland, USA.

Connexin $43(\mathrm{Cx} 43)$ is the major protein component of gap junctions that electrically couple cardiomyocytes at the intercalated disc. Oxidant stress, reduced $C_{x} 43$ expression, and altered subcellular localization are present in many forms of structural heart disease. These changes in $\mathrm{Cx} 43$ lead to alterations in electrical conduction in the ventricle and predispose to lethal cardiac arrhythmias. In their study in this issue of the JCI, Smyth et al. tested the hypothesis that oxidant stress perturbs connexon forward trafficking along microtubules to gap junctions (see the related article beginning on page 266). Failing human ventricular myocardium exhibited a reduction in $\mathrm{Cx} 43$ and the microtubule-capping protein EB1 at intercalated discs. Oxidant stress in the adult mouse heart reduced $\mathrm{N}$-cadherin, EB1, and $\mathrm{Cx} 43$ colocalization. In HeLa cells and neonatal mouse ventricular myocytes, peroxide exposure displaced EB1 from the plus ends of microtubules and altered microtubule dynamics. Mutational disruption of the EB1-tubulin interaction mimicked the effects of oxidant stress, including a reduction in surface $\mathrm{Cx} 43$ expression. These data provide important new molecular insights into the regulation of $\mathrm{Cx} 43$ at gap junctions and may identify targets for preservation of cellular coupling in the diseased heart.

Rapid propagation of electrical impulses in excitable tissue is essential to processes as diverse as cognition, movement, and the genesis of the heartbeat. Central to rapid conduction in the heart and other organs are gap junctions. Gap junctions are low-resistance conduits between cells,

Conflict of interest: The author has received a grant from Boston Scientific Inc.

Citation for this article: J. Clin. Invest. 120:87-89 (2010). doi:10.1172/JCI41780. comprised of proteins called connexins. In the heart, connexins are key mediators of electrical conduction and are thus central to excitation and contraction. Connexins hexamerize to form connexons or hemichannels in the membranes of apposing cells that dock head-to-head to form intact gap junction channels (Figure 1).

The major connexin of working ventricular myocardium is connexin 43 (Cx43). Cx43 is richly endowed with protein interaction domains and sites of phosphorylation that contribute to regulation of the functional expression of gap junction channels. The carboxyl terminus contains a PDZ-binding domain, multiple consensus serine and tyrosine phosphorylation sites, and binding sites for tubulins. Post-translational modifications and protein-protein interactions are thought to be important for proper formation and localization of clusters of gap junctions into so-called "plaques," although Cx43 with a truncated carboxyl terminus forms working gap junction channels (1).

\section{Structural complexity of intercalated discs}

The cardiomyocyte is a complex and highly structured cell. In normal myocardium, gap junction channels are prominently located at intercalated discs positioned at cell ends, mediating electrical propagation that is preferentially in the direction of the long axis of the cell (Figure 1). A remarkable feature of gap junctions and other ion channels is their extremely short half-life (approximately 1-3 hours) $(2,3)$. The short half-life of gap junction channels is particularly surprising given the transmembrane topology and the complex structure of the intercalated disc that houses connexin-containing gap junctional plaques and implies highly coordinated and tightly regulated trafficking mecha- 